\title{
The Three-Dimensional Structure of Giardia lamblia Virus and its Comparison to Other Members of the Family Totiviridae
}

\author{
M. E. Janssen ${ }^{1}$, K. N. Parent ${ }^{2}$, Y. Takagi ${ }^{3}$, M. L. Nibert ${ }^{3}$, T. S. Baker ${ }^{1,4}$ \\ ${ }^{1}$ Department of Chemistry and Biochemistry, University of California San Diego, La Jolla, CA, USA \\ ${ }^{2}$ Department of Biochemistry and Molecular Biology, Michigan State Univ., East Lansing, MI, USA \\ ${ }^{3}$ Department of Microbiology and Immunobiology, Harvard Medical School, Boston, MA, USA \\ ${ }^{4}$ Division of Biological Sciences, University of California San Diego, La Jolla, CA, USA
}

Giardia lamblia is a flagellated protozoan parasite that causes giardiasis, which is an infection of the small intestine. It is the most common cause of water-borne, parasitic illness in the US [1]. G. lamblia itself is susceptible to infection by a small, double stranded RNA (dsRNA) virus, called Giardia lamblia virus (GLV), which in turn is thought to influence the severity of giardiasis caused by its host [2]. GLV is a member of the family Totiviridae that is distinct for its non-segmented dsRNA genomes with overlapping open reading frames and isometric virions [2]. A resemblance of GLV to other family members has not been demonstrated at the structural level, nor has the location of the RNA dependent RNA polymerase inside the particle been established yet. We have determined the 3D structure of GLV by cryo-electron microscopy and icosahedral image reconstruction to an estimated resolution limit of 7.2 $\AA$, and compared its structure to other totiviruses, such as Trichomonas vaginalis virus 1 (TVV1), Saccharomyces cerevisiae virus L-A (ScV-L-A), and Helminthosporium victoria virus 190S (HvV190S), to help us understand the basic biology and evolution of these related viruses.

Purified virions from $G$. lamblia were prepared for cryo-electron microscopy via standard, rapid freezeplunge procedures [4]. Micrographs were recorded on Kodak SO-163 electron image film at $200 \mathrm{kV}$ in an FEI Polara microscope under minimum-dose conditions $\left(\sim 25 \mathrm{e} / \mathrm{A}^{2}\right)$ at a nominal magnification of $\mathrm{x} 59,000$ and with defocus levels ranging from 0.7 to $3.3 \mu \mathrm{m}$. A total of 8,959 individual particle images were extracted from 71 digitized micrographs (1.09- $\AA$ pixels at the specimen). An initial reconstruction at $\sim 29$ - $\AA$ resolution was generated from 150 particle images using the random-model procedure [5], which subsequently served as a starting model to initiate full orientation and origin determinations of the entire set of images using AUTO3DEM [6]. The Segger v1.7 tool in the Chimera visualization package [7] was used to segment the final map and to examine interactions between subunits [8].

The 3D map of GLV reveals a so-called, ' $T=2$ ' capsid consisting of 60 asymmetric dimers, each of which contains two chemically identical monomers that occupy non-identical environments. A channel, $\sim 15 \AA$ in diameter at the 5-fold axis, is large enough to permit exchange of nucleoside triphosphates and newly synthesized RNA transcripts. Unlike what is observed in many other dsRNA viruses, the packaged genome of GLV is not visualized as a series of concentric rings. The overall morphologies of the capsid and the individual GLV subunits are very similar to those of TVV1 [9] and ScV-L-A [10]. Shared features between representative family members of Totiviridae suggest a common ancestor. Though transmission of most mature totivirus virions generally occurs intracellularly via cell division, sporogenesis, or cell fusion, GLV is unique because it can infect virus-free G. lamblia isolates [3]. Also, the diameter of GLV (485- $\AA$ ) is larger than that of other totiviruses whose structures are known. Lastly, the capsid protein of GLV (886 aa) is significantly larger than the capsid proteins of TVV1 (678 aa), ScV-L-A (680 aa), and HvV190S (772 aa), which may mean that the additional residues in GLV are surface-exposed and play a role in the extracellular transmission of GLV. 


\section{References}

[1] B. Lebwohl, R.J. Deckelbaum, and P.H.R. Green, Gastrointest Endosc. 57 (2003) 906

[2] R. L. Miller, A. L. Wang and C. C. Wang, Mol. Biochem. Parasitol. 28 (1988) 189

[3] J. H. Tai et al., Virology 216 (1996) 124

[4] T. S. Baker, N. H. Olson and S. D. Fuller, Microbiol. Mol. Biol. Rev. 63 (1999) 862

[5] X. Yan et al., J. Struct. Biol. 157 (2007) 211

[6] X. Yan et al., J. Struct. Biol. 157 (2007) 73

[7] E. F. Pettersen et al., J. Comput. Chem. 25 (2004) 1605

[8] S. Dunn et al., PLOS Path. (2013) In press

[9] K. N. Parent et al., mBio (2013) Submitted

[10] H. Naitow et al., Nat. Struct. Biol. 9 (2002) 725

[11] Funding was provided by NIH grants 1S10 RR020016 and R37 GM033050 to TSB, and by UCSD and the Agouron Foundation to TSB.
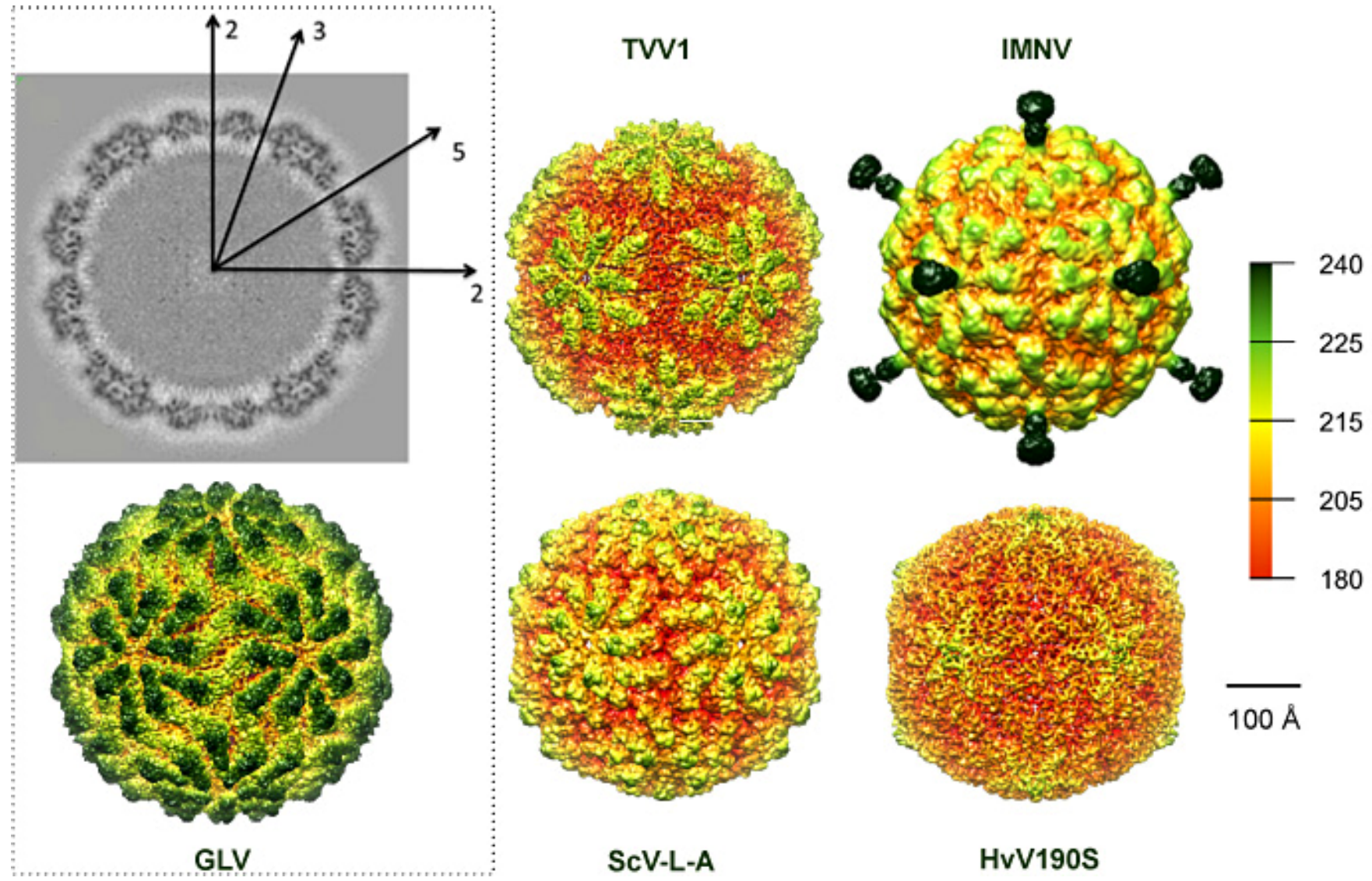

Fig.1. Comparison of GLV 3D structure with four other totiviruses. Upper left: central (equatorial), one pixel $(1.09 \AA)$ thick section through the virion density map shown in gray-scale (white and black correspond to lowest and highest densities, respectively). Icosahedral 2-, 3-, and 5-fold symmetry axes are marked. Lower left: space-filling view of the GLV particle surface as viewed down a 2-fold axis and color-coded by radius according to the color bar on the right (radii given in $\AA$ ). Center and right columns: four other totiviruses, all viewed along a 2-fold axis and rendered with the same color mapping scheme. 